\title{
Maduración in vitro de ovas de Brycon henni (Pisces: Characidae) con dos preparaciones hormonales
}

\author{
In vitro maturation of Brycon henni eggs (Pisces: Characidae) with two \\ hormonal preparations
}

\author{
Juan David Montoya Páez ${ }^{1,3}$, Hermes Pineda Santis ${ }^{1}$, Giovanni Restrepo-Betancur ${ }^{2}$
}

\section{Resumen}

La sabaleta (Brycon henni) es una especie de interés comercial en Colombia. Una de las principales barreras para los procesos de conservación de esta especie es la asincronía temporal entre los ciclos reproductivos de las hembras y los machos, que podría ser solucionado mediante el uso de procesos biotecnológicos. El objetivo de esta investigación fue evaluar la maduración in vitro de ovas de sabaleta (Brycon henni) mediante el uso de dos preparaciones hormonales. Un total de 1200 ovas fueron sometidas a maduración in vitro en presencia de extracto de hipófisis de carpa (EPC), hormona liberadora de gonadotropinas de salmón (sGnRHa) y un control sin estimulación hormonal. Las ovas se incubaron durante 2 horas a $25^{\circ} \mathrm{C}$ en agitación orbital constante y $80 \%$ de $\mathrm{O}_{2}$. Se evaluó la maduración in vitro de las ovas mediante la clasificación de la posición del núcleo en cuatro categorías: migratorio, central, atrésico y periférico. Se encontraron resultados superiores de avance de la maduración por la presencia de núcleo migratorio $(\mathrm{p}<0.05)$ para EPC $(46.0 \pm 8.4 \%)$ y sGnRHa $(40.0 \pm 13.3 \%)$, en comparación con el control $(24.0 \pm 9.6 \%)$. Se concluye que el uso de las preparaciones hormonales EPC y sGnRHa durante la maduración in vitro promueve el avance de la maduración de las ovas de sabaleta (Brycon henni).

Palabras clave: hormonas; maduración in vitro; peces; reproducción

\footnotetext{
${ }^{1}$ Facultad de Ciencias Agrarias, Politécnico Colombiano Jaime Isaza Cadavid, Medellín, Colombia

${ }^{2}$ Facultad de Ciencias Agrarias, Universidad Nacional de Colombia, Medellín, Colombia

${ }^{3}$ E-mail: jdmontoya@elpoli.edu.co
}

Investigación financiada por la Dirección de Investigación y Posgrados del Politécnico Colombiano Jaime Isaza Cadavid

Recibido: 2 de febrero de 2018

Aceptado para publicación: 18 de julio de 2018 
The 'sabaleta' (Brycon henni) is a species of commercial interest in Colombia. One of the main barriers to the conservation processes of this species is the temporal asynchrony between the reproductive cycles of females and males, which could be solved using biotechnological processes. The aim of this investigation was to evaluate the in vitro maturation of 'sabaleta'eggs (Brycon henni) using two hormonal preparations. A total of 1200 eggs were subjected to in vitro maturation in the presence of carp pituitary extract (EPC), salmon gonadotropin-releasing hormone ( $\mathrm{sGnRHa}$ ) and a control without hormonal stimulation. The eggs were incubated for 2 hours at $25^{\circ} \mathrm{C}$ in constant orbital shaking and $80 \% \mathrm{O}_{2}$. The in vitro maturation of the eggs was evaluated by classifying the position of the nucleus in four categories: migratory, central, atretic and peripheral. Higher maturation advancement were obtained due to the presence of migratory nucleus $(p<0.05)$ for EPC $(46.0 \pm 8.4 \%)$ and sGnRHa $(40.0 \pm 13.3 \%)$, compared to the control $(24.0 \pm 9.6 \%)$. It is concluded that the use of EPC and sGnRHa hormonal preparations during in vitro maturation promotes the advancement of maturation of 'sabaleta' eggs (Brycon henni).

Key words: hormones; in vitro maturation; fishes; reproduction

\section{INTRODUCCIÓN}

La sabaleta Brycon henni (Eigenmann, 1913) es una especie nativa de peces de la familia Characidae, presente en los pequeños ríos que nacen en la cordillera central de Colombia (Dahl, 1971) y con subpoblaciones distribuidas en los ríos Cauca, Magdalena, San Juan, Dagua y Patía (Montoya-López et al., 2006; Pineda-Santis et al., 2007). Esta especie es endémica, en especial en la cuenca de los ríos Porce y sus afluentes como el Rio Grande, Nus, San Rafael y San Carlos, todos localizados en la zona de embalses del departamento de Antioquia; siendo tal vez la especie euritérmica más representativa, puesto que se encuentra en aguas limpias y transparentes con temperaturas entre los $18 \mathrm{y}$ $28{ }^{\circ} \mathrm{C}$ y concentraciones de oxígeno disuelto entre 6 y $10 \mathrm{mg} / 1$ (Lenis et al., 2009). En el ámbito reproductivo, se han descrito diversos aspectos de la gametogénesis y la reproducción del género Brycon (Arboleda et al., 2005; Gonçalves et al., 2006; Montoya-Lopéz 2006). Como peces teleósteos, sus ovarios muestran una estructura general similar a los mamíferos, como órganos pares alargados y orientados longitudinalmente dentro de la cavidad abdominal (Legac et al., 1993). Los ovarios están compartimentados por septos formados por pliegues del epitelio germinal (laminillas ovígeras) (Jalabert, 2005). Durante la etapa de diferenciación del folículo primario en el huevo, el oocito dentro del folículo ovárico, adquiere la capacidad de formar un embrión viable después de la fertilización (Lubzens, 2010).

El desarrollo de los oocitos se puede dividir en cinco etapas, las cuales son similares entre todos los peces teleósteos: crecimiento primario, estado de alveolos corticales, estado de desarrollo vitelogénico temprano, estado de desarrollo vitelogénico tardío y maduración/ovulación (Leino et al., 2005; Urbatza, 2011). El término maduración de oocitos designa una serie de procesos celulares complejos, que corresponden a la reanudación de la meiosis hasta el segundo arresto en la metafase (Jalabert et al., 1991). En la ovulación, los ovocitos maduros son liberados de su folículo en la cavidad ovárica, antes de ser puestos afuera a través del oviducto y de la papila genital (Kobajashi et al., 2008). La finalización de la segunda división meiótica del 
ovocito maduro se produce solo después de la fertilización evidenciando la formación de un óvulo haploide y un cuerpo polar (Urbatza, 2011).

Se conoce que la maduración de los gametos de los peces puede requerir, según la especie, de la estimulación mediante condiciones ambientales como temperatura y salinidad del agua, del uso de estimulación hormonal o de la combinación de ambos (Muller et al., 2005; Kucharczyk et al., 2017). Se han utilizado preparados hormonales con extracto de hipófisis de carpa (EPC) y análogos de GnRH como la hormona liberadora de gonadotropinas de salmón (sGnRHa), combinados con inhibidores o antagonistas de la dopamina (Ovopel, Ovaprim, OvaRH), los cuales actúan como bloqueadores de los factores inhibidores de la liberación de gonadotropinas (GRIF) (Peter et al., 1988), influencian directamente las gónadas y causan la maduración de los oocitos (Kucharczyk et al., 1997, Brzuska, 2006; Jamróz et al., 2008). También se han realizado estudios con estimulación hormonal con gonadotropina coriónica humana (hCG) (Kucharczyk et al., 1997; 2017). Kucharczyk et al. (2017) reportaron que la aplicación de un tratamiento con sGnRHa en burbot (Lota lota L) sincronizó la maduración final de los ovocitos dando como resultado una alta tasa de supervivencia de los embriones. La estimulación de hembras Brycon henni con EPC, Ovopel y Ovaprim influyó en el diámetro de los huevos obtenidos y en su fertilización (Lenis et al., 2009). En otros estudios de inducción hormonal de la especie se reporta el uso de hipófisis de carpa, hipófisis de Bufo marinus, LH-RH y hCG (Granados et al., 1986; Zapata y Vanegas, 1993).

La estimulación hormonal para la maduración de los gametos en peces se realiza directamente sobre los animales, de manera que son menos los reportes de maduración in vitro de las ovas. Milla et al. (2006) implementaron un protocolo de maduración in vitro de oocitos en folículos ováricos de
Oncorhynchus mykiss mediante el uso de esteroides, encontrando que el rompimiento de la vesícula germinal (GVBD) solo se presentó después de la estimulación con 17 $\alpha, 20 \beta-$ dihidroxi-4-pregnen-3-ona (DHP). En otros estudios se reporta que el tratamiento de folículos ováricos con IGF-I e insulina, aumenta la concentración de la hormona inductora de la maduración DHP en el medio y que IGF-I, IGF-II y b-insulina inducen GVBD en oocitos desnudos (Mukherjee et al., 2006).

La maduración de las ovas puede determinarse mediante la posición de su núcleo, a través de criterios previamente definidos como: vesícula germinal en la posición central (CGV, etapa 1), migración temprana de la vesícula germinal (MGV, etapa 2), migración tardía de la vesícula germinal (más que la mitad del radio - PGV, etapa 3) y vesícula germinal periférica o rompimiento de la vesícula germinal (GVBD, etapa 4) (Brzuska, 1979; Kucharczyk et al., 2005; Targoñska et al., 2015). Es así que la madurez de las ovas se expresa a través de la migración de la vesícula germinal (Targoñska et al., 2015). Estudios en Brycon henni evaluaron las ovas considerando igualmente la posición del núcleo y la presencia de ovocitos atrofiados o deformes (Lenis et al., 2009). El objetivo de este trabajo fue evaluar el efecto del extracto de hipófisis de carpa (EPC) y la hormona liberadora de gonadotropinas de salmón (sGnRHa) en la maduración in vitro de ovas de sabaleta (Brycon henni).

\section{Materiales y Métodos}

\section{Localización}

El material de investigación (ovas de Brycon henni) fue recolectado en la granja experimental piscícola Jhon Jairo González del Politécnico Colombiano Jaime Isaza Cadavid, ubicada en el municipio de San Jerónimo (Antioquia, Colombia). Las muestras fueron procesadas y evaluadas en la granja 
experimental y en el Laboratorio de Biotecnología Animal del Centro de Experimentación y Laboratorios del Politécnico Colombiano Jaime Isaza Cadavid, Sede Be1lo (Antioquia, Colombia). Los animales estuvieron sometidos a condiciones estándar de alimentación, ambiente, manejo general y reproductivo.

\section{Colecta de Ovas}

Los peces se manejaron según las directrices publicadas para la experimentación de peces en las Buenas Prácticas en la Producción Acuícola (ICA, 2007). Se utilizaron 20 hembras Brycon henni, las cuales fueron anestesiadas por inmersión en solución de benzocaína ( $1 \mathrm{~g} / 1$ de agua). Los peces se retiraron del estanque cuando se observó la pérdida del eje de nado y se secó cuidadosamente la papila urogenital para evitar el contacto de las ovas con el agua. Inmediatamente después se procedió a extraer una muestra de ovas de cada hembra, introduciendo un catéter plástico por el oviducto. Luego las ovas se almacenaron en un dispositivo de transporte y se llevaron hasta el laboratorio a temperatura ambiente en un tiempo máximo de tres horas.

\section{Maduración in vitro}

Las ovas se sometieron por separado durante $2 \mathrm{~h}$ a un medio basado en TCM 199 (Gibco ${ }^{\circledR}$, EEUU), suplementado con dos preparaciones hormonales: extracto de hipófisis de carpa (EPC, Acuagranja, Colombia) u hormona liberadora de gonadotropinas de salmón (sGnRHa, Ovaprim, Syndel, EEUU) a razón de $0.1 \mathrm{mg} / \mathrm{ml} \mathrm{y} 0.1 \% \mathrm{v} / \mathrm{v}$, respectivamente. Se incluyó un grupo control sin suplementación hormonal. Se tomaron 300 ovas por tratamiento distribuidas en grupos de 10 ovas y fueron puestas en cajas de Petri con $4 \mathrm{ml}$ de medio suplementado, bajo condiciones de incubación a $25{ }^{\circ} \mathrm{C}$ en agitación orbital constante a $100 \mathrm{rpm}$ (Thermo Scientific $^{\circledR}$, EEUU) y condiciones atmosféricas, de acuerdo a un protocolo modificado del descrito por Milla et al. (2006).

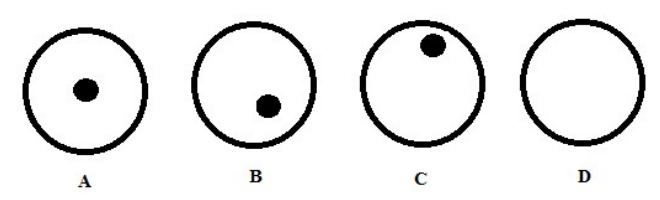

Figura 1. Diagrama de la posición del núcleo en las ovas. A) Central, B) Migratorio, C) Periférico, D) Atrésico

La evaluación de la maduración in vitro de las ovas se realizó según la posición de su núcleo, que fue clasificado en cuatro categorías: migratorio, central, periférico o atrésico; considerando criterios reportados por Brzuska (1979) y Lenis et al. (2009) (Figura 1). Los huevos fueron aclarados para eliminar la interferencia del vitelo en la observación del núcleo, siendo sometidos a una solución compuesta por $30 \%$ de formol, $30 \%$ de ácido acético y $40 \%$ de alcohol (v/v) durante $2 \mathrm{~min}$. Se consideraron como ovas en proceso de maduración aquellas que presentaron la migración de la vesícula germinal (núcleo migratorio) según lo descrito en investigaciones previas (Kucharczyk et al., 2005; Targoñska et al., 2015). La presencia de yemas de coloración blanquecina o heterogénea indicó la no maduración de las ovas (Beltrán et al., 1978).

\section{Análisis Estadístico}

Se empleó un diseño completamente al azar. Se ajustó un modelo lineal generalizado para determinar el efecto de las fuentes de variación (grupo de ovas, fecha de recolección y tratamiento) sobre la maduración de las ovas de acuerdo con la posición del núcleo (variable dependiente). Se evaluó la normalidad de los datos mediante una prueba de Kolmogorov-Smirnov. Para la comparación de las medias entre los tratamientos se utilizó la prueba de Duncan. Todos los análisis fueron realizados mediante el programa SAS v. 9.2 (SAS Inst. Inc., Cary, NC). 
Cuadro 1. Distribución de ovas de Brycon henni según la posición del núcleo después de la maduración in vitro

\begin{tabular}{lccccc}
\hline $\begin{array}{l}\text { Posición del } \\
\text { núcleo }\end{array}$ & Media (\%) & DE (\%) & CV (\%) & Máximo & Mínimo \\
\hline Migratorio & 36.7 & 14.0 & 38.1 & 60 & 10 \\
Central & 23.0 & 11.2 & 48.6 & 40 & 0 \\
Atrésico & 35.3 & 19.8 & 55.9 & 80 & 0 \\
Periférico & 2.0 & 4.8 & 242.1 & 20 & 0 \\
\hline
\end{tabular}

Resultados obtenidos a partir de 10 grupos de ovas sometidas a maduración in vitro por tratamiento (10 ovas por grupo). DE: desviación estándar; CV: coeficiente de variación

Cuadro 2. Distribución de ovas de Brycon henni según la posición del núcleo después de la maduración in vitro por tratamiento

\begin{tabular}{lcccc}
\hline \multirow{2}{*}{ Tratamiento } & \multicolumn{4}{c}{ Posición del núcleo } \\
\cline { 2 - 5 } & Migratorio (\%) & Central (\%) & Atrésico (\%) & Periférico (\%) \\
\hline Control & $24.0 \pm 9.7^{\mathrm{a}}$ & $17.0 \pm 11.6^{\mathrm{a}}$ & $49.0 \pm 23.8^{\mathrm{a}}$ & $2.0 \pm 4.2^{\mathrm{a}}$ \\
EPC & $46.0 \pm 8.4^{\mathrm{b}}$ & $25.0 \pm 10.8^{\mathrm{ab}}$ & $26.0 \pm 15.0^{\mathrm{b}}$ & $3.0 \pm 6.7^{\mathrm{a}}$ \\
sGnRHa & $40.0 \pm 13.3^{\mathrm{b}}$ & $27.0 \pm 9.5^{\mathrm{b}}$ & $31.0 \pm 12.0^{\mathrm{b}}$ & $1.0 \pm 3.2^{\mathrm{a}}$ \\
\hline
\end{tabular}

Resultados obtenidos a partir de 30 grupos de ovas sometidas a maduración in vitro (10 ovas por grupo). Los resultados se presentan como media \pm desviación estándar Promedios con letras diferentes denotan diferencia significativa $(p<0.05)$ entre tratamientos EPC: extracto de hipófisis de carpa; sGnRHa: hormona liberadora de gonadotropinas de salmón

\section{Resultados}

A partir de la maduración in vitro y la evaluación de la posición del núcleo de cada ova como indicador del estado de maduración se encontró prevalencia de núcleos migratorios, atrésicos y centrales, en este mismo orden. Así mismo, se observó una reducida proporción de núcleos periféricos y una alta variabilidad en la proporción de presentación de estos últimos (Cuadro 1).

Los efectos incluidos en los modelos estadísticos ajustados explicaron en una media a baja proporción la maduración in vitro de las ovas según la posición del núcleo, con valores para el coeficiente de determinación
$\left(\mathrm{R}^{2}\right)$ de $0.50,0.43,0.43$ y 0.27 para núcleo migratorio, central, atrésico y periférico, respectivamente. Los efectos fijos de la fecha de colecta y el tratamiento fueron significativos en el modelo ajustado para la evaluación de núcleos atrésicos $(p<0.05)$. En el modelo ajustado para explicar la variabilidad en la presencia de núcleos migratorios solo fue significativo el efecto del tratamiento $(\mathrm{p}<0.05)$. No se encontraron efectos significativos en los modelos ajustados para núcleos centrales y periféricos.

Se observaron diferencias entre los tratamientos (control, EPC y sGnRHa) de acuerdo con la posición del núcleo de las ovas, cuando estos fueron catalogados como migratorios, centrales y atrésicos $(p<0.05)$ 
(Cuadro 2). Para los núcleos migratorios, el uso de las hormonas EPC y sGnRHa resultó en una tasa superior de ovas en proceso de maduración (migración de la vesícula germinal) en comparación con el tratamiento control $(\mathrm{p}<0.05)$. De otro lado, se observó un efecto inverso de ambas hormonas sobre la presentación de núcleos atrésicos, con una reducción significativa respecto al control $(\mathrm{p}<0.05)$. La maduración in vitro de las ovas en presencia de sGnRHa produjo una proporción superior de ovas con núcleo central respecto al control $(\mathrm{p}<0.05)$; sin embargo, no se observó diferencia significativa entre ambas preparaciones hormonales. La suplementación hormonal no generó diferencias en la presencia de núcleos periféricos, lo cual pudo ser influenciado por la reducida proporción de los mismos para todos los tratamientos.

\section{Discusión}

La sabaleta Brycon henni es una especie endémica de las zonas de montaña de Colombia, que se adapta bien a las aguas templadas y cálidas, con la cual desde hace más de tres décadas se ha venido trabajando en varios aspectos de su biología, ecología y reproducción en confinamiento (Lenis et al., 2015). Es catalogada como una especie promisoria para la cría en cautiverio y para el repoblamiento, siendo importante para la seguridad alimentaria de las poblaciones ribereñas de las zonas que habita (MontoyaLópez et al., 2006). Sin embargo, existe la necesidad de buscar alternativas para la recuperación de esta especie, muy apetecida por la calidad de su carne, lo que ha provocado un descenso considerable en sus índices de captura (Lenis et al., 2009).

Se reporta que existe un posible asincronismo entre los picos de madurez de hembras y machos a lo largo del año (Lenis et al., 2015). En los sistemas de producción piscícola no ha sido todavía posible romper la estacionalidad del ciclo reproductivo (Cruz-
Casallas et al., 2006), siendo por esta razón, que se utilizan hormonas para inducir la madurez y la ovulación, las cuales no son suficientes si no van acompañadas de condiciones ambientales específicas, tales como la temperatura y el fotoperiodo (Kucharczyk et al., 2005, 2017).

Se han desarrollado trabajos de inducción hormonal como alternativa para generar la maduración de ovas de Brycon henni. En un estudio de Lenis et al. (2009), la estimulación de las hembras con EPC, Ovopel y Ovaprim influyó en el diámetro de los huevos obtenidos y en su fertilización. En el presente estudio donde se indujo la maduración de las ovas en condiciones in vitro, se evaluó su estado de maduración mediante la posición del núcleo. En otros estudios con peces del género Brycon se ha considerado la migración de la vesícula germinal como un signo de avance en la maduración final de las ovas. Pardo et al. (2006) realizaron la inducción de hembras de yamú Brycon amazonicus con EPC, encontrando un progreso en la migración de la vesícula germinal con $6.2 \pm 3.2 \%$ en estado migrando, $74.9 \pm$ $5.3 \%$ maduro y $18.85 \pm 4.9 \%$ en estado atrésico.

En el presente estudio, la EPC y la sGnRHa indujeron la migración de la vesícula germinal, toda vez que se observó un incremento significativo de ovas con núcleo migratorio que coincidió con una menor proporción de ovas atrésicas (Cuadro 2). Incluso se observó un incremento de ovas con núcleos con posición central, que fue significativo al menos para el tratamiento con sGnRHa. Sin embargo, la proporción de ovas con núcleos periféricos correspondientes a la maduración final o GVBD fue bastante reducida, sin diferencia alguna por efecto del tratamiento. Lo anterior, podría interpretarse como un efecto positivo de ambos preparados hormonales en la inducción de la migración de la vesícula germinal, como indicador del avance de la maduración; sin embargo, el efecto de la EPC y la sGnRHa, o las condi- 
ciones utilizadas en la maduración in vitro, como el tiempo de incubación, el medio utilizado o la agitación durante la incubación, pudieron no ser las necesarias para inducir la maduración final (GVBD). Por otro lado, la reducción de la proporción de ovas atrésicas puede considerarse un evento positivo para la obtención de un gran número de huevos con alta supervivencia durante las primeras etapas de desarrollo, como un evento crucial para la sostenibilidad y el éxito de la acuicultura (Lubzens et al., 2017).

En el caso de la sabaleta, se recomiendan realizar inducciones hormonales exógenas en las hembras cuando el porcentaje de oocitos con núcleo en estado migratorio es superior al 70\%. De igual manera, un tamaño de la ova entre 1.5 y $2.0 \mathrm{~mm}$ se constituye también como un elemento para evaluar su madurez (Lenis et al., 2015).

De otro lado, no se conocen otros trabajos donde se reporte la maduración in vitro de ovas de sabaleta utilizando hormonas de forma exógena como la EPC y la sGnRHa; sin embargo, para la carpa común Cyprinus carpa, se reporta el uso de IGF-I, IGF-II y b-insulina para la maduración de folículos ováricos y oocitos desnudos con altas tasas de GVBD (Mukherjee et al., 2006). De otro lado, Milla et al. (2006) implementaron un protocolo de maduración in vitro de oocitos en folículos ováricos de Oncorhynchus mykiss mediante el uso de esteroides, encontrando que el rompimiento de la vesícula germinal (GVBD) solo se presentó después de la estimulación con 17 $\alpha, 20 \beta$-dihidroxi-4pregnen-3-ona (DHP).

De acuerdo con los resultados obtenidos y a la escasa información sobre la maduración in vitro de ovas de Brycon henni y otras especies, se hace necesario evaluar otras alternativas, como el uso de diferentes preparaciones hormonales, acompañadas de condiciones adecuadas de incubación, considerando factores ambientales habituales para el desarrollo y la maduración de las ovas.

\section{Conclusión}

El uso de preparaciones hormonales de extracto de hipófisis de carpa (EPC) y hormona liberadora de gonadotropinas de salmón (sGnRHa) durante la maduración in vitro promueve el avance de la maduración (migración de la vesícula germinal) de las ovas de sabaleta (Brycon henni), convirtiéndose en una alternativa para obtención de ovas que puedan ser útiles para los procesos de fertilización fuera de las temporadas reproductivas de la especie.

\section{Literatura Citada}

1. Arboleda L, Olivera MA, Tabares CJ, Echeverri A, Serna DF. 2005. Madurez gonadal en hembras de sabaleta (Brycon henni) y su relación con variables medioambientales. Rev Politécnica 1:95-103.

2. Beltrán CN, Beltrán I, Sierra R. 1978. Notas preliminares sobre la reproducción y cultivo de la sabaleta (Brycon henni, Eigenman 1913). Bogotá, Colombia: Instituto de Recursos Naturales. 27 p.

3. Brzuska E. 1979. The in vivo method of estimating the stages of oocytes maturation in carp Cyprinus carpio L. Acta Hydrobiologica 21: 423-433.

4. Brzuska E. 2006. Artificial spawning of female Lithuanian strain B carp (Cyprinus carpio L) after treatment with carp pituitary homogenate, Ovopel or [D-Tle ${ }^{6}$, ProNHEt9] GnRH-a (Lecirelin). Aquac Res 37: 264-271. doi: 10.1111/j.1365-2109. 2005.01428.x

5. Cruz-Casallas PE, Medina-Robles VM, Velasco-Santamaría YM. 2006. Protocolo para la crioconservación de semen de yamú (Brycon amazonicus Spix \& Agassiz 1829). Rev Colomb Cienc Pec 19: 146-151 
6. Dahl G 1971. Los peces del norte de Colombia. Bogotá, Colombia: Inderena. $391 \mathrm{p}$.

7. Gonçalves TL, Bazzoli N, Brito MFG. 2006. Gametogenesis and reproduction of the matrinxã Brycon orthotaenia (Günther, 1864) (Pisces: Characidae) in the São Francisco river, Minas Gerais, Brazil. Braz J Biol 66: 513-522. doi: 10.1590/S1519-69842006000300018

8. Granados H, Sarria A, Galvis D, López J, Ortiz J. 1986. Reproducción inducida en Brycon henni Eigenmann, 1913 (Sabaleta) Rhamdia wagneri Gunther (Barbudo). Rev Vet Zoot Caldas 5: 68-91.

9. Jalabert B, Fostier A, Breton B, Weil C. 1991. Oocyte maturation in vertebrates. In: Pang PKT, Schreibman MP (eds). Vertebrate endocrinology: funda-mentals and biomedical applications. Part A. San Diego, California: Academic Press. p 23-90.

10. Jalabert B. 2005. Particularities of reproduction and oogenesis in teleost fish compared to mammals. Reprod Nutr Dev 45: 261-279. doi: 10.1051/ rnd:2005019

11. Jamróz M, Kucharczyk D, Hakua$B^{3} a_{6}$ owska A, Krejszeff $S$, Kujawa $R$, Kupren K, et al. 2008. Comparing the effectiveness of Ovopel, Ovaprim and LH-RH analogue used in the controlled reproduction of ide, Leuciscus idus (L). Arch Polish Fisheries 16: 363-370. doi: 10.2478/s10086-008-0024-2

12. [ICA] Instituto Colombiano Agropecuario. 2007. Buenas prácticas en la producción acuícola: directrices sanitarias y de inocuidad para la producción acuícola destinada al consumo humano. Bogotá: Produmedios. 65 p. [Internet]. Disponible en: http://www.cib.-uaem.mx/ pdf/buenas_practicas.pdf

13. Kobayashi T, Kajiura-Kobayashi H, Guan G, Nagahama Y. 2008. Sexual dimorphic expression of DMRT1 and Sox9a during gonadal differentiation and hormone-induced sex reversal in the teleost fish Nile tilapia (Oreochromis niloticus). Dev Dynam 237: 297-306. doi: 10.1002/dvdy.21409.

14. Kucharczyk D, Kujawa R, Luczynski M, Glogowski J, Babiak I, Wyszomirska E. 1997. Induced spawning in bream, Abramis brama (L), using carp pituitary extract and hCG. Aquac Res 28: 139-144. doi: 10.1046/j.1365-2109.1997.t01-1-00840.x

15. Kucharczyk D, Kujawa R, Mamcarz A, Targoñtska-Dietrich K, Wyszomirska E, Glogowski J, Babiak I, Szabó T. 2005. Induced spawning in bream (Abramis brama $\mathrm{L}$ ) using pellets containing GnRH. Czech J Anim Sci 50: 89-95. doi: 10.17221/4000-CJAS

16. Kucharczyk D, Nowosad J, Kujawa R, Dietrich G, Biegaj M, Sikora M, Luczynski MJ. 2017. Comparison of spontaneous and hormone-induced reproduction of burbot (Lota lota $\mathrm{L}$ ) under hatchery conditions. Aqualculture 485: 25-29. doi: 10.1016/j.aquaculture.2017.08.040

17. Legac F, Blaise O, Fostier A, Lebail PY, Loir M, Mourot B, Weil C. 1993. Growth Hormone (GH) and reproduction: a review. Fish Physiol Biochem 11: 219-232. doi: 10.1007/BF00004569

18. Leino RL, Jensen KM, Ankley GT. 2005. Gonadal histology and characteristic histopathology associated with endocrine disruption in the adult fathead minnow (Pimephales promelas). Environ Toxicol Phar 19: 85-98. doi: 10.1016/j.etap.2004.05.010

19. Lenis G, Restrepo L, Cruz-Casallas P. 2009. Evaluación de tres protocolos de tratamiento hormonal sobre el diámetro de ovocitos de sabaleta Brycon henni. Rev Colomb Cienc Pec 22:131-142.

20. Lenis GA, Cruz PE, David Ruales CA. 2015. Reproducción inducida de la sabaleta Brycon henni: revisión bibliográfica. Rev Lasallista Invest 12: 211220.

21. Lubzens E, Bobe J, Young G, Sullivan CV. 2017. Maternal investment in fish oocytes and eggs: The molecular cargo 
and its contribution to fertility and early development. Aquaculture 472: 107-143. doi: 10.1016/j.aquaculture.2016.10.029

22. Lubzens E, Young G Bobe J, Cerda J. 2010. Oogenesis in teleosts: how eggs are formed. Gen Comp Endocr 165: 365387. doi: 10.1016/j.ygcen.2009.05.022

23. Milla S, Jalabert B, Rime H, Prunet P, Bobe J. 2006. Hydration of rainbow trout oocyte during meiotic maturation and in vitro regulation by $17,20 \beta$ dihydroxy-4-pregnen-3-one and cortisol. J Exp Biol 209: 1147-1156. doi: 10.1242/ jeb.02094

24. Montoya-López A, Carrillo LM, Olivera-Ángel M. 2006. Algunos aspectos biológicos y del manejo en cautiverio de la sabaleta Brycon henni Eigenmann, 1913 (Pisces Characidae). Rev Colomb Cienc Pec 19: 180-185.

25. Muller T, Baska F, Niklesz, C, Horn P, Váradi B, Bercsényi M. 2005. The testis histology of artificially maturated European eel (Anguilla anguilla L) at the end of sexual maturation, and spermatozoa ultrastructure in freshwater rearing. Acta Biol Hung 56: 169-172. doi: 10.1556/ABiol.56.2005.1-2.17

26. Mukherjee D, Mukherjee D, Sen U, Paul S, Bhattacharyya SP. 2006. In vitro effects of insulin-like growth factors and insulin on oocyte maturation and maturation-inducing steroid production in ovarian follicles of common carp, Cyprinus carpio. Comp Biochem Physiol 144: 63-77. doi: 10.1016/ j.cbpa.2006.01.012

27. Pardo-Carrasco SC, Arias-Castellanos JA, Suárez-Mahecha $\mathrm{H}$, CruzCasallas PE, Vásquez-Torres W,
Atencio-García V, Zaniboni-Filho E. 2006. Inducción a la maduración final y ovulación del Yamú Brycon amazonicus con EPC y mGnRH-a. Rev Colomb Cienc Pec 19: 160-166.

28. Peter RE, Lin HR, Van Der Kraak G 1988. Induced ovulation and spawning of cultured freshwater fish in China: advances in application of GnRH analogues and dopamine antagonists. Aquaculture 74: 1-10. doi: 10.1016/00448486(88)90080-4

29. Pineda-Santis H, Arboleda L, Echeverry A, Urcuqui S, Pareja D, Olivera M, Builes J. 2007. Caracterización de la diversidad genética en el pez Brycon henni (Characiformes: Characidae) en Colombia central por medio de marcadores RAPD. Rev Biol Trop 55: 1025-1035. doi: 10.15517/rbt.v55i34.5975

30. Targoñska K, Kupren K, Kujawa R, Mamcarz A, Kaczkowski $Z$, Glogowski J, et al. 2015. Artificial reproduction of different dace, Leuciscus leuciscus (L) populations as a method for biodiversity preservation. Turk J Fish Aquat Sc 15: 477-485. doi: 10.4194/1303-2712-v15_2 34

31. Urbatzka R, Rocha MJ, Roche E. 2011. Regulation of ovarian development and function in teleost. In: Hormones and reproduction of vertebrates. Amsterdam: Elsevier. p 65-81

32. Zapata OL, Vanegas PR. 1993. Aspectos importantes sobre la reproducción inducida y el metabolismo de las gónadas de la sabaleta Brycon henni. Tesis de Biólogo. Medellín, Colombia: Univ. de Antioquia. $67 \mathrm{p}$. 